\title{
A TETHERED UPPER STAGE FOR SMALL LAUNCHERS - PRELIMINARY ANALYSIS, DESIGN, AND TEST
}

\author{
A. Hyslop, E. J. van der Heide, M. Kruijff, C. Bonnal, \\ C. Talbot, and A. Espinosa
}

The use of mechanical tethers for momentum transfer is a space-proven concept and leads to many possible applications. One such application is launch-assist. By tethering a payload to a reaction mass and executing a controlled deployment and tether-cut, the momentum of the reaction mass can be sacrificed to obtain a boost for the payload. In this way, after a launcher has delivered its payload to a low elliptic orbit, a tether deployment can be used to circularize the payload and simultaneously de-orbit the spent stage. This paper discusses the results of the design study and hardware testing of the deployment.

\section{ACRONYMS}

$\begin{array}{ll}\text { AUTEC } & \text { Autonomous Tether Cutter } \\ \text { c.o.m. } & \text { center of mass } \\ \text { GPS } & \text { Global Positioning System } \\ \text { IMU } & \text { Inertial Measurement Unit } \\ \text { LEO } & \text { Low Earth Orbit } \\ \text { MTBSim } & \text { Monte Carlo Tether Bead Simulator } \\ \text { OBC } & \text { On-Board Computer } \\ \text { OLD } & \text { Optical Loop Detector } \\ \text { RAAN } & \text { Right Ascension of the Ascending Node } \\ \text { REST } & \text { Re-Entry Simulator Tool } \\ \text { RTBSim } & \text { Real-time Tether Bead Simulator } \\ \text { s.m.a. } & \text { semimajor axis } \\ \text { SSO } & \text { Sun Synchronous Orbit }\end{array}$

\section{INTRODUCTION}

Mechanical tethers, through the action of momentum transfer, can potentially be used as the upper stage of a launch system. By deploying a tether between 
a mission payload and a spent stage (reaction mass), subsequent cutting of the tether will place the mission payload in a higher orbit and the spent stage in a lower one. This study investigated a tethered upper stage that is able to raise and circularize the orbit of a mission payload, correct for the solid stages' dispersion error, and simultaneously de-orbit a spent solid stage. The launcher designation convention used is: $\mathrm{Pxx}$ ( $\mathrm{xx}$ tons of solid propellant) and Tyy (yy km of deployable tether). The title of the study is therefore "T-Series." Since the proposed T-Series tethered end-mass is somewhat large compared to many past demo missions [1], and the tether length, deployment velocity, and tensions involved are much higher, it is of interest to see if typical tether deployer hardware is capable of accurately performing the deployment. A deployer test rig was developed for the YES2 (the Second Young Engineers' Satellite.) [2] to evaluate the deployer hardware and software in real time, and this test rig is adaptable for testing any deployer system [3]. Since the tension levels are two orders of magnitude larger for T-Series compared to YES2, it was decided that another hardware-in-the-loop test was required to make a better judgement regarding the transferability of YES2/SEDS* hardware to T-Series.

This paper describes the theory behind the T-Series concept, the capabilities of the design, and a description of the mission and system hardware. The design of the tether deployment trajectories will be discussed, and Monte Carlo simulations used to determine the expected mission success rate. Finally, the T-Series design is weighed against rival concepts and the results of hardware testing are presented.

\section{LAUNCH SYSTEM SUMMARY}

The T-Series family of launchers (Fig. 1a) uses low-cost solid propellant and a simple tether deployer (integrated with the upper stage structure) to provide reliable affordable access to Low Earth Orbit (LEO), without leaving space debris. The solid stage achieved orbit (errors) are compensated by the tether deployment.

The nominal case presented here is for a 600-kilometer sun-synchronous circular target orbit with a fictitious P9-P9-P2 solid stage configuration and a desired mission payload of $100-150 \mathrm{~kg}$; so, one is interested in a tethered upper stage for a rather small next generation launcher. Assuming a Sun Sunchronous Orbit (SSO) and a 175-kilometer perigee for the transfer orbit, Fig. $1 b$ shows the deliverable satellite payload vs. circular target altitude for the benchmark T-Series configuration. Naturally, the performance curve can be raised further by increasing solid propellant mass or specific impulse. Target orbits of alternate eccentricity and inclination are also possible (restricted only by capability of solid stages).

${ }^{*}$ SEDS - Small Expendable-tether Deployer System. 


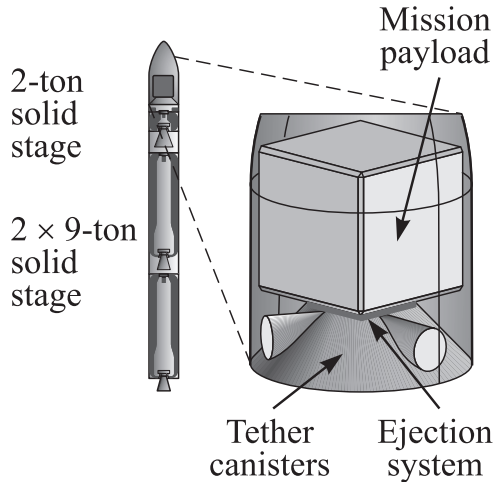

(a)

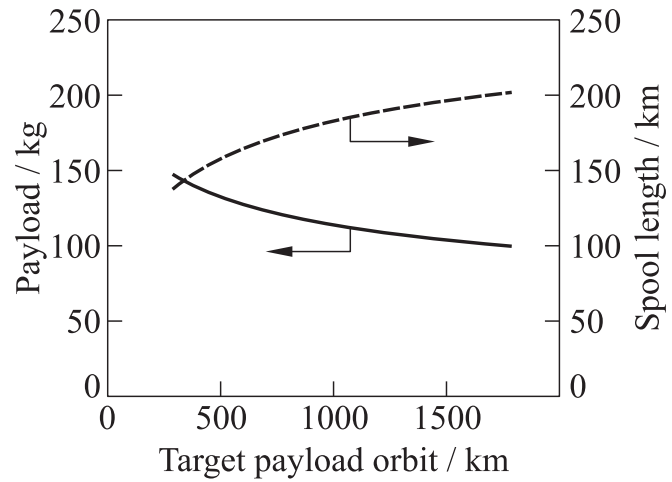

(b)

Figure 1 (a) T-Series launcher (insert shows tether upper stage), and (b) P9-P9-P2Tx launcher feasibility map assuming 175-kilometer perigee for the transfer orbit. Estimated reliability: 97\%; accuracy ( $3 \sigma$ error): eccentricity $=0.00195$, s.m.a. $=11.6 \mathrm{~km}$, RAAN $=0.01^{\circ}$, and inclination $=0.06^{\circ}$

\section{TETHER THEORY}

\subsection{Momentum Exchange Principles}

Consider a scenario beginning with two point masses (payload and spent stage) initially in the same orbit, connected by a tether. To induce some initial separation, an ejection system is employed to send the payload upward and the spent stage downward. The resulting motion causes the tether to unreel from its storage canister.

Although the ejection initiates the tether deployment, the deployment would halt due to hardware friction forces in the absence of the gravity gradient. This refers to the fact that for tethered masses, gravity is slightly stronger at the lower mass compared to the upper mass. Also, because the tether is constraining both masses to follow the same orbit, there is a large centrifugal force on the upper mass (which would otherwise be travelling slower without the tether) and a smaller centrifugal force on the lower mass (which is travelling faster than it would without the tether). With the centrifugal force on the upper mass being greater than its gravity, it tends to pull upwards on the tether. With the reverse situation on the lower mass, the tether is pulled downward, thus creating overall tension. Because the net centrifugal-gravity forces act along the Earth-pointing vector, there is always a moment-couple tending to restore the tethered system to the vertical orientation. If the tether is fixed at both ends, this effect drives the motion (libration) of the system [4]. 
Because the tether is not fixed at the lower end, it can be pulled off its spool by having tension in the adjacent section of tether. Thus, if the tether tension is greater than the friction in the deployer hardware, the tether will come off the spool and deploy. As the tether gets longer, the gradient forces become stronger and the deployment accelerates at an increasing rate. Due to the changing length of the tether, the equations of motion reveal acceleration terms that tend to move the system away from the vertical orientation. This is the Coriolis effect and it will generally cause the tether to form a large in-plane angle with the vertical so long as the deployment speed is reasonably high.

Once the desired length is reached, the deployer must execute a braking action to stop the tether deployment and the fixed-length tether will swing back to the vertical - in absence of Coriolis acceleration. If the tether is cut as it passes through the vertical, then: $(i)$ prograde (or retro if required) velocity is imparted to the payload from the swing, and $(i i)$ the orbital path constraint is removed thus leaving the payload exposed to a higher altitude orbit with the speed corresponding to a lower orbit (defined by the tethered-system center of mass (c.o.m.)). These effects combine to raise the perigee of the payload's orbit, whilst simultaneously lowering the perigee of the spent stage. If the delta- $\mathrm{V}$ is big enough, the spent stage can be reentered into the atmosphere.

\subsection{Terminology}

Required payload delta- $\mathbf{V}$ refers to the difference between the speed of the tethered system c.o.m. prior to cut and the speed corresponding with a circular orbit at the target altitude. Tethered systems exhibit an important advantage over conventional propulsive systems in that the payload is already at the required circular orbit height by tether deployment. If a rocket system were to boost from $150 \times 560$ to $600 \times 600$, it would require a burn at perigee to raise the apogee to $600 \mathrm{~km}$ then another delta- $\mathrm{V}$ at apogee to circularize. This two-burn maneuver of $138 \mathrm{~m} / \mathrm{s}$ delta- $\mathrm{V}$ is replaced by a tether deployment (40-kilometer altitude gain for payload) and cut (95 m/s delta-V) for a T60 system (Fig. 2).

With regards to maintaining a clean orbit and thereby re-entering the spent stage, the rocket system would require an additional delta- $\mathrm{V}$ of about $-60 \mathrm{~m} / \mathrm{s}$, whilst the tether does this without any additional effort.

Reference refers to the deployment profile(s) that is(are) designed before the mission and stored on the On-Board Computer (OBC). The OBC attempts to control the deployment to track the most appropriate reference profile.

Zero-error refers to the case where the solid launcher delivers its intended orbit with zero error. Typically, this term pertains specifically to zero error in the delivered apogee.

Mission payload is payload that will be delivered into the target orbit. This does not include deployer or tether mass.

Total payload $=$ Mission payload + tether deployer + tether mass. 


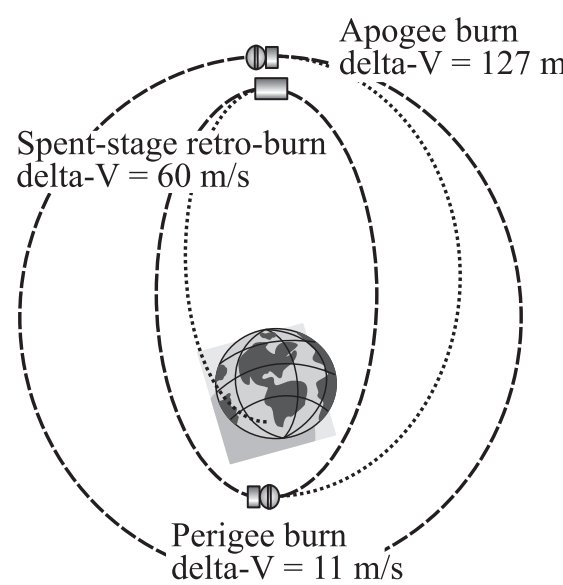

Conventional Hohmann transfer total payload delta- $\mathrm{V}$ required $=138 \mathrm{~m} / \mathrm{s}$

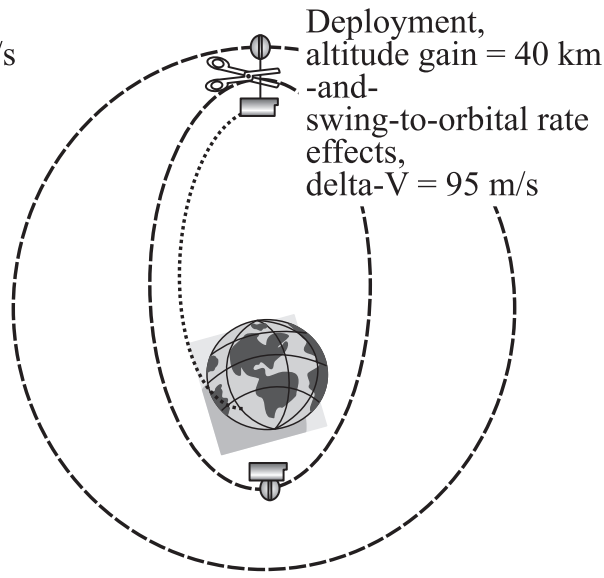

Tethered momentum transfer total payload delta- $\mathrm{V}$ required $=95 \mathrm{~m} / \mathrm{s}$

Figure 2 Rocket delta-V vs. tether delta-V

\section{MISSION DESCRIPTION}

The launch begins with 3 solid stages delivering the spent stage into an orbit of $\sim 175 \times 542 \mathrm{~km}$ (example for 600-kilometer target orbit) (Fig. 3). An Inertial Measurement Unit / Global Positioning System (IMU/GPS) measures the achieved orbit - the apogee altitude in particular. An OBC, with 10 stored reference profiles, selects the appropriate payload ejection time, how much tether to deploy (100 km in this instance), what deployment profile to follow, and when to release the payload from the tethered system. The spent stage attitude control system points the mission payload toward zenith.

At ejection time, springs are released sending the payload upwards at $\sim 1.3 \mathrm{~m} / \mathrm{s}$. The payload is attached to a tether, which begins to deploy from spool \# 1. The deployer controls the tension to follow the predefined deployment profile. After spool \# 1 is depleted, unwinding begins from spool \# 2 via a simple coupling system. The fully deployed tether swings back to the vertical orientation, also coinciding with apogee pass, and a timer on the payload activates a pyro tether cutter. The payload is released into a circular orbit of $600 \mathrm{~km}$. The tether, attached to the spent stage, burns up and the stage itself burns or fragments land $7500 \mathrm{~km}$ west (see Fig. 9) of the launch site.

Before the mission, the cut-time and launch time must be fixed to align with the celestial Right Ascension of the Ascending Node (RAAN) requirement. There must also be enough time in orbit to perform all possible tether deployment profiles (whose selection depends on solid-stage-delivered orbit). Even for an 


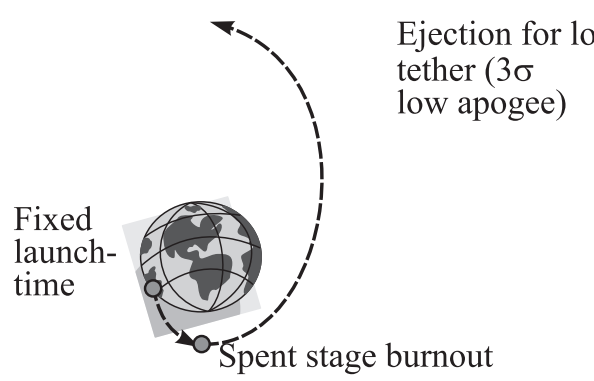

(a)

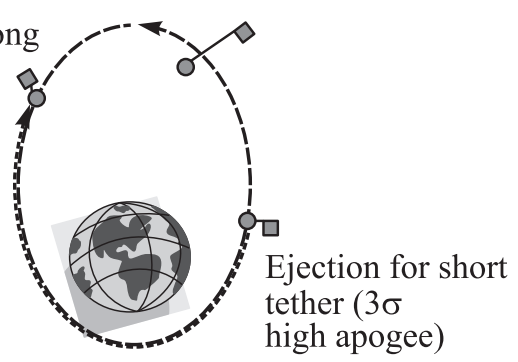

(b)

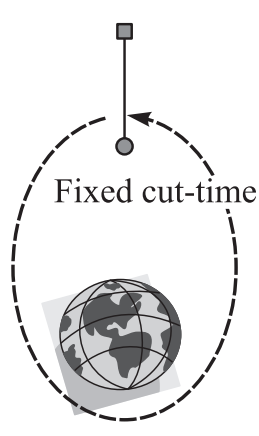

(c)

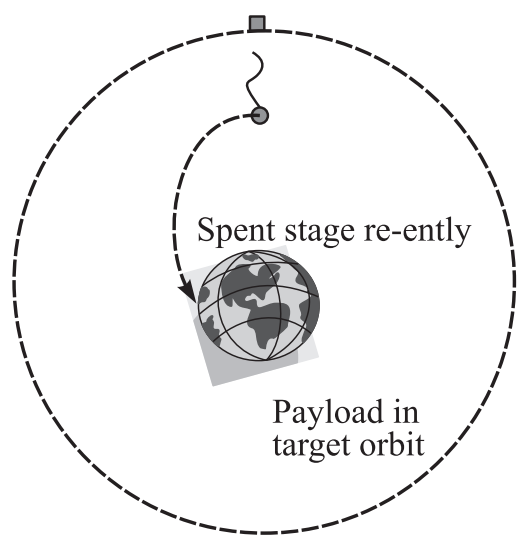

(d)

Figure 3 Mission plan: (a) launch, 0-0.5 orbits; (b) 0.5-1.5 orbits (ejection); (c) 2.5 orbits (cut); and $(d)$ reentry

SSO, the target RAAN drifts with time and can therefore still be achieved at an earlier or later time by altering the cut and launch time. This allows flexibility for changing, prior to takeoff, the fragments dispersion area of the spent stage by cutting at a time when the Earth is in a different orientation. The fragments dispersion area is principally dependent on the preplanned time in orbit and is then fixed relative to the launch site. The choice of payload celestial RAAN requirement does not alter this.

\section{LAUNCHER CONCEPTUAL DESIGN}

For a fixed solid launcher configuration, the three main design variables for a tether-assisted launch are the mission payload mass, tether length, and perigee/ apogee of transfer orbit. The relevant tradeoffs are: 
- Payload mass:

- high payload mass means reduced feasibility window (reduced dispersion range of initial conditions from which a circular orbit is still attainable) but steeper re-entry angle;

- Tether length:

- there is a minimum tether length (and therefore, maximum P2delivered apogee) below which the delta- $\mathrm{V}$ required can no longer be supplied by swing motion;

- the deployed tether length should be selected based on the P2delivered apogee altitude, and the cut should always be made at apogee pass. Away from the apogee, the orbital velocity has a large radial component, and local-plane tether motion is insufficient to cancel this; and

- if the true anomaly at ejection is near perigee, when the tether is still short, air drag may push the system to a dangerously high in-plane angle;

- P2 burnout perigee:

- high P2-delivered perigee means increased feasibility window but shallower re-entry;

- the lower limit for perigee is approximately $140 \mathrm{~km}$, below which air drag causes significant bending of the tether shape - thereby reducing behavioral predictability; and

- thermal issues can occur for long tethers passing relatively low perigees; and

- P2 burnout apogee:

- low apogee altitudes require a very long tether, which leads to large airdrag during perigee pass. This air drag can reduce the semimajor axis of the system by tens of kilometers. Then even more tether needs to be deployed to make up for the altitude lost; and

- since high apogee altitudes require relatively short tethers, there exists a maximum P2 burnout apogee above which the tether cannot deliver the delta- $\mathrm{V}$ required.

The process of selecting a feasible configuration was conducted as follows:

1. Tether sizing: dividing the tether into 3 composite sections, it was decided that the 1st section should be $0.4 \mathrm{~mm}$ Dyneema, the $2 \mathrm{nd}-0.3 \mathrm{~mm}$ Zylon, and the 3rd $-0.35 \mathrm{~mm}$ Zylon. These selections comply with strength, thermal limit, and survivability requirements whilst also minimizing mass. 
2. The delta- $\mathrm{V}$ required for circularization depends on both $\mathrm{P} 2$-delivered perigee and payload mass (since it affects the center of mass of the tethered system). Aiming for minimum tether length, which corresponds with highest possible apogee, one has to equate required delta- $\mathrm{V}$ with available delta- $\mathrm{V}$ (a function of tether length and payload) and solve for the transfer orbit apogee altitude.

3. With known $+3 \sigma$ apogee and known solid launcher dispersions, one then knows the lowest possible apogee. This corresponds with the maximum tether length required (to physically position the satellite in the target orbit) and a check must be made to ensure that the particular configuration does not result in the lower end of the tether passing too close to the atmosphere during deployment.

4. The spool length determined above leads to a total upper stage system mass, which must be checked against what is deliverable by the solid stages.

5. Checks 3 and 4 are combined to give a feasibility map as a function of mission payload and P2-delivered perigee.

For the 600-kilometer target orbit with assumed launcher dispersions of -32/ $+24 \mathrm{~km}$ at $3 \sigma$, the above procedure resulted in the following design:

$\begin{array}{lr}\text { Mission payload, kg } & 127 \\ \text { P2-burnout perigee, km } & 175 \\ \text { Zero-error apogee, km } & 542 \\ 3 \sigma \text { apogee undershoot, km } & 510 \\ 3 \sigma \text { apogee overshoot, km } & 566 \\ \text { Spool length (for } 3 \sigma \text { apogee undershoot), km } & 159 \\ \text { Zero-error case - deployed tether, km } & 81 \\ 3 \sigma \text { apogee overshoot - deployed tether, km } & 47 \\ \text { Total upper stage }+ \text { mission payload } & 160 \\ \text { P9-P9-P2 liftable } & 161\end{array}$

\section{HARDWARE DESIGN}

The subsystems breakdown, by physical location, is given below.

Deployer: SEDS-type "barberpole" friction brake, tether canister(s), ejection system. Figure 4 shows this hardware as built for the YES2 satellite (Fig. 5). The same hardware could be adapted for T-Series.

Launcher: OBC, telemetry unit, orbit determination system, attitude control system, power supply system.

Payload interface: Autonomous tether cutter (AUTEC), ejection aystem interface. 


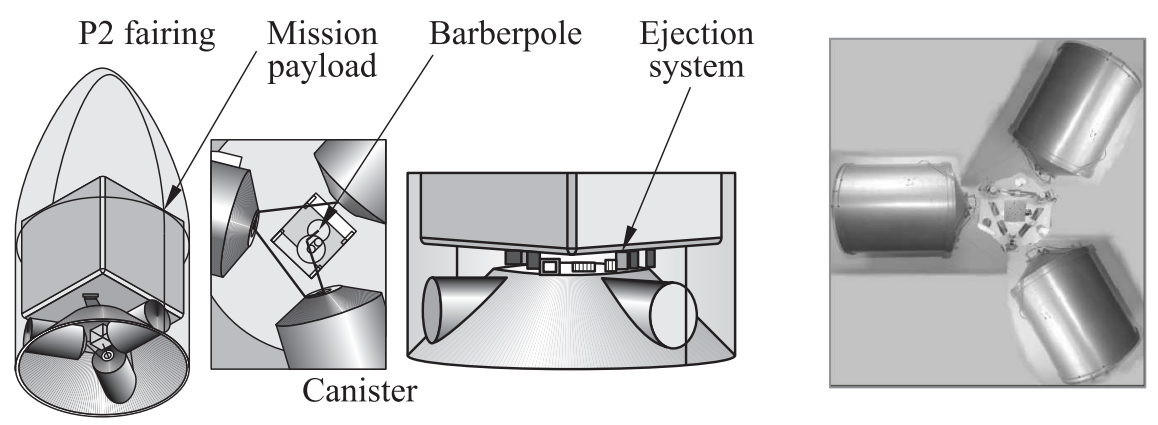

Figure 4 Conceptual hardware layout on P2 stage, artists impression of coupled canisters

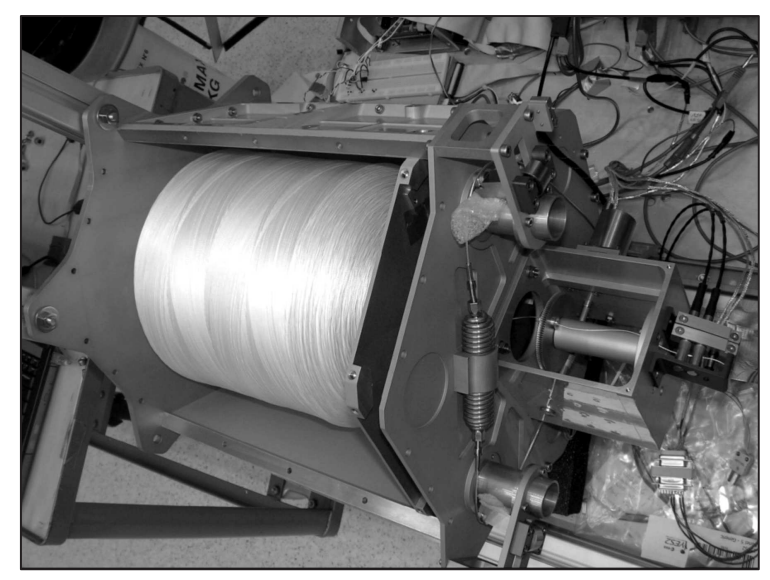

Figure 5 YES2 tether deployment system, featuring tether in canister, ejection system, "barberpole" friction brake, and tether cutter assembly

The AUTEC allows for a cut-time to be preprogrammed into it before ejection via a releasable connector. The AUTEC has on-board batteries which activate a pyro tether cutter, which releases the payload into a new orbit. In this way there is no communication necessary with the payload after the cut-time has been programmed.

It was decided that multiple canisters were required to house the 159kilometer tether due to volume constraints and the necessity to distribute the launch loads. The end-tether of each spool is secured to the canister wall with Kapton and second surface mirror tape and then threads out the exit hole and connects with the subsequent canister/spool. When a spool is fully unwound, 
the tether becomes taught and rips the Kapton thus freeing itself to allow commencement of unwinding from the next spool.

The system mass estimate for the tether upper stage is $35 \mathrm{~kg}$, with a maximum of 14-watt power consumption.

\section{TETHER TRAJECTORY}

\subsection{Launch and Ejection Time}

Although the optimum algorithm would be to have on-board computation of trajectories based on measured orbital parameters, it will be assumed here that a discrete number of tether deployment trajectories are stored on-board in table format and that each corresponds with a small range of P2-delivered apogees. This method is simpler and did not appear to lead to greater injection inaccuracy. Figure 6 shows the results of the reference file design exercise in a mission timeline. The shortest possible mission duration was used. Any increase in duration, to alter $\mathrm{P} 2$ fragment dispersion area, would have to be in multiples of an orbital period to maintain the cut time at apogee.

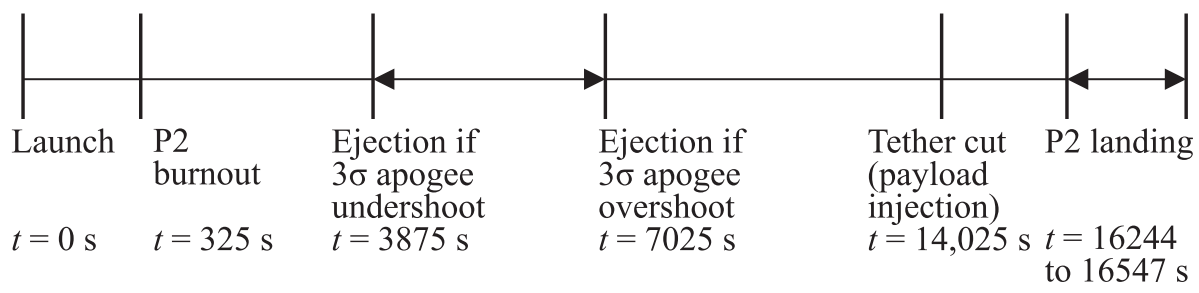

(a)

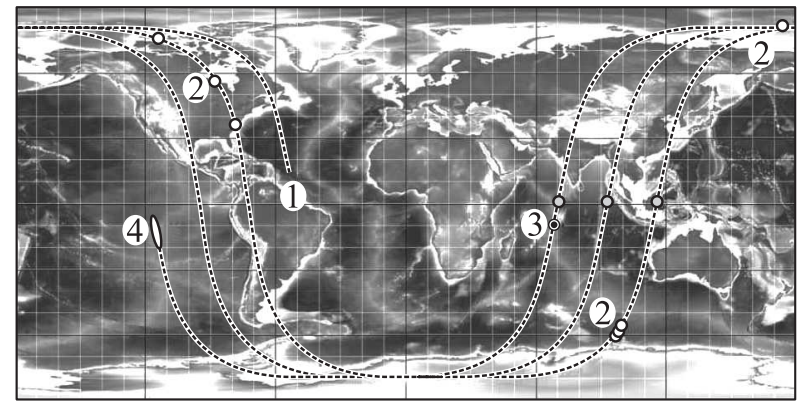

(b)

Figure 6 (a) Mission timeline; and (b) ground track 
The ejection times were calculated by an iterative process whereby deployer tension (brake turns), ejection time, and target tether length were altered to achieve the correct final orbit. Essentially, target tether length tunes the altitude at release, ejection time tunes the timing of the swing through vertical with apogee pass, and the brake turns tunes the delta- $\mathrm{V}$ delivered to the payload.

From Fig. $6 b$, note the following events:

1. Launch in Kourou, French Guiana.

2. Payload ejection, start of tether deployment (location depends on reference file selected).

3. Tether cut, payload separation from tethered system.

4. Fragment dispersion area for P2 spent stage.

\subsection{Deployment Profile}

The zero-apogee-error case and the profiles for the two most extreme apogee error cases are illustrated in Fig. 7.

From Fig. 7, it can be seen that the cases of higher P2-delivered apogee require longer tethers and back swing (to cancel out some of the delta- $\mathrm{V}$ from
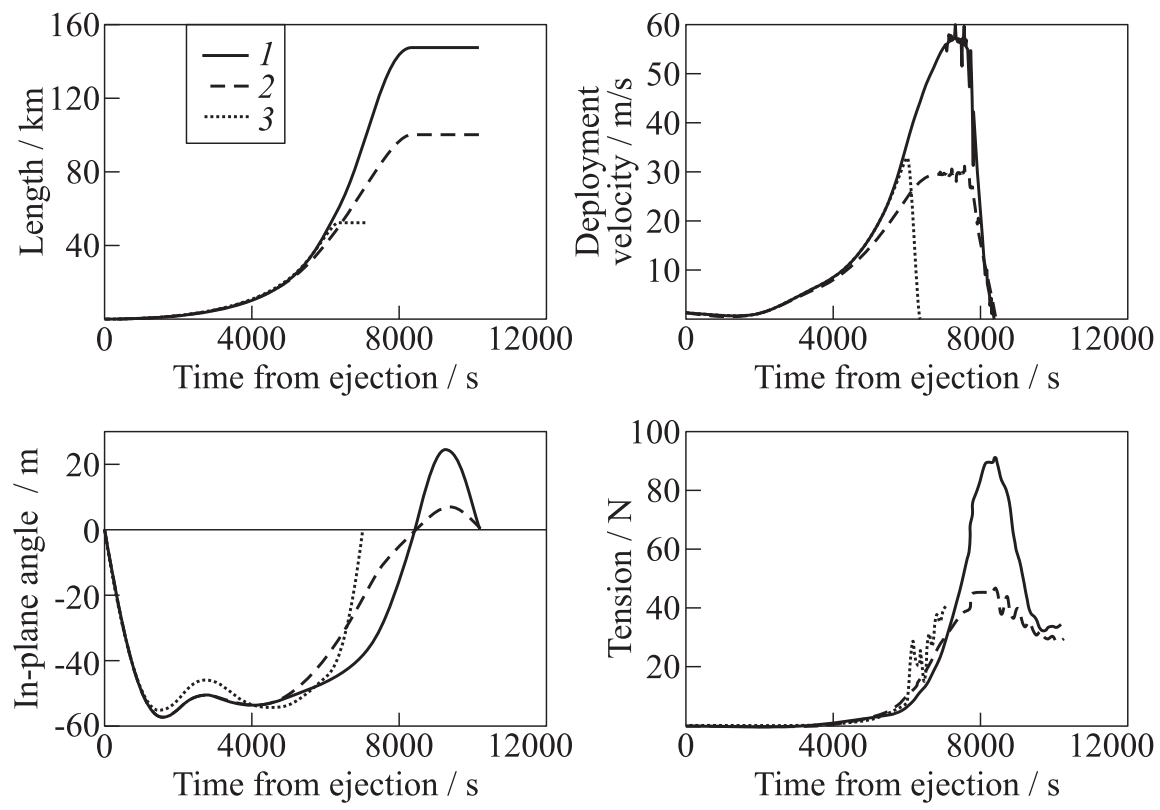

Figure 7 Reference profiles (only extremes and zero-error cases are displayed). P2 burnout apogee: $1-514.5 \mathrm{~km} ; 2-542.0$; and $3-564.0 \mathrm{~km}$ 
the tethered radial arm effect). The need for back swing also dictates a much longer deployment profile; therefore, the ejection time must be much earlier. The substantial fluctuations in the tension graphs is indicative of the fact that the profiles are not optimized - deemed beyond the scope of this study.

\section{MONTE CARLO SIMULATIONS}

The Monte Carlo Tether Bead Simulator (MTBSim) developed by Delta-Utec models the tether as a series of lumped masses connected by spring-damper systems [5]. The following models were switched on for the T-Series simulations: deployer friction modeling, Optical Loop Detector (OLD) length measurement noise, barberpole friction noise, OBC discrete controller time, finite barberpole stepper speed, selection of deployment reference profile based on initial orbit, tether temperature (heating from free molecular flow, planetary radiation, albedo, sun radiation), NASA standard atmosphere, solar pressure and reflection model (Lambertian), J2 gravity effect. For adequate capture of tether flexibility, 50 elements (beads) were used in the simulations.

The Re-Entry Simulator Tool (REST) simulates the aerothermodynamics of re-entry [6]. For T-Series, the attitude motion of the spend stage was not considered. The Spent stage is modeled as a sphere, but with the same ballistic coefficient and comparable drag/lift as specified by the CNES launcher specification (as used in launch simulations). As a first approximation, the entry assumes no spent stage fragmentation (empty, sturdy, filled with thermal protection, rigid body).

The results of the Monte Carlo are shown in Fig. 8 indicating that the accuracy of the integrated launch system is favorable.

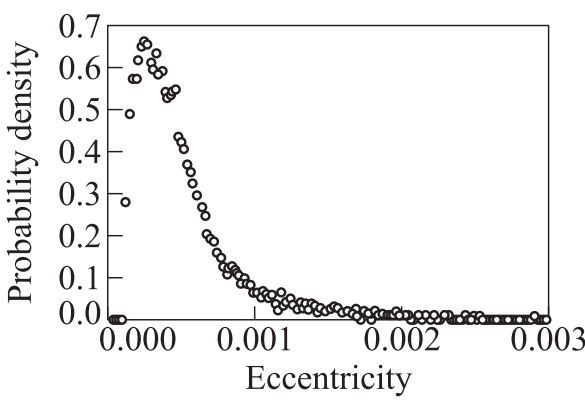

(a)

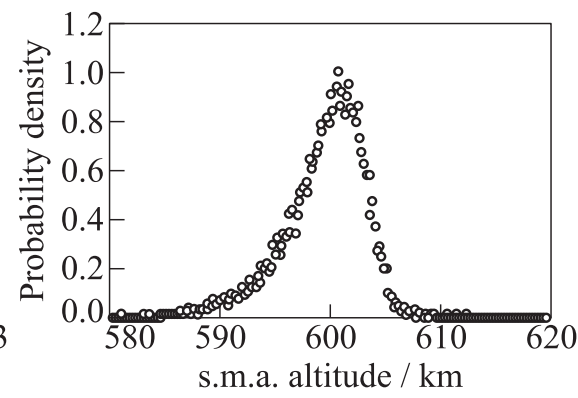

(b)

Figure 8 Distribution curves for final payload orbit eccentricity $(a)$ and semimajor axis (s.m.a.) altitude $(b)$ 


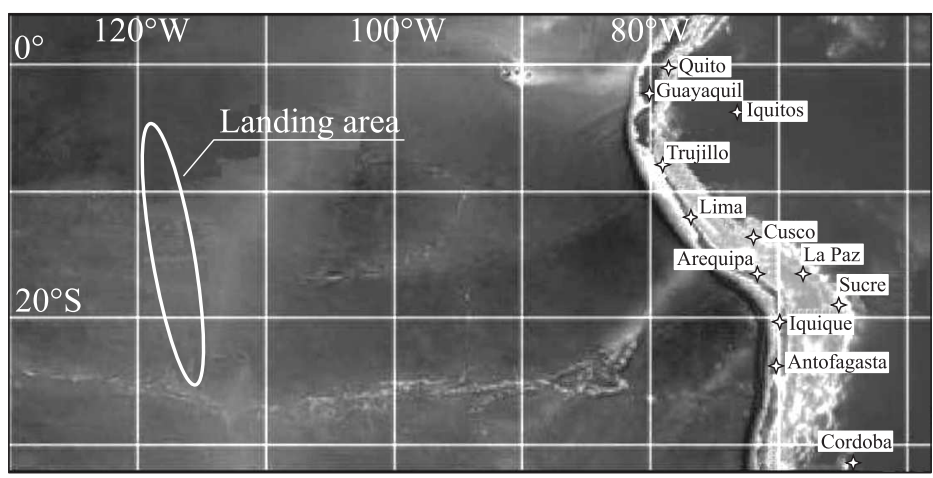

Figure 9 P2 spent stage $3 \sigma$ dispersion ellipse

The estimated spent stage entry angle is about $-1.78^{\circ} \pm 0.13^{\circ}$, which results in the following landing ellipse (Fig. 9). Due to the relatively high perigee and the relatively low end-mass ratio, the entry angle is quite shallow.

\section{COMPARISON TO ALTERNATIVES}

A quick comparison can be done with three more classical configurations (note that the goal is to reach the same payload performance and to have no launcher debris in orbit as in the tether scenario).

The first option is to force the P2 stage to reach the final orbit (the T159 is removed) with a more vertical trajectory and a longer coast phase. In such a case, the performance is far below the objective, the accuracy is not guaranteed, and an additional device for deorbiting is necessary.

The second option is the replacement of the T159 by a classical (4th) propulsive stage, whose function is to circularize the payload and then deorbit itself after separation. In that case, the transfer orbit of the P2 must be lowered to ensure a natural and controlled reentry of it (perigee altitude around $0 \mathrm{~km}$ ). The same performance can then be obtained with a L0.02 stage (20 kg of hydrazine) weighing a total mass of $50 \mathrm{~kg}$ (including autonomous avionics), or a L0.03 (less than $30 \mathrm{~kg}$ of hydrazine) weighing a total mass of $100 \mathrm{~kg}$ (including the avionics for the entire launcher, removed from the initial P2 stage).

The third option is to replace the P2 and T159 by a unique liquid upper stage (storable propellant) capable of 3 ignitions (to reach successively the transfer orbit, final circular orbit and then deorbiting). An L2 (2 t of $\left.\mathrm{N}_{2} \mathrm{O}_{4} / \mathrm{MMH}\right)$ can reach the same performance considering classical technologies, but with a higher cost and less reliability. 


\section{DEPLOYMENT TESTING}

\subsection{Test Rig Architecture}

Without a means to directly simulate the gravity and centrifugal gradient (which enables the tether to deploy passively in space), a computer-based simulator is used to determine the effect of these forces on the deployment velocity with the additional input of applied tension from the rig (Fig. 10). The updated velocity value is fed back to the rig and the tether is consequently 'pulled' out of the canister at this speed. After deployment, the tether is then recovered for reuse $[7]$.

The basic events of the test are as follows:

1. Thirty two kilometers of tether (scaled down from $100 \mathrm{~km}$ - see comment in subsection 10.2.2) is wound on a core and placed in the canister.

2. The test commences by the pulling motor simulating the ejection of the payload (an initial jerk to a $0.4 \mathrm{~m} / \mathrm{s}$ deployment — scaled down from $1.33 \mathrm{~m} / \mathrm{s})$.

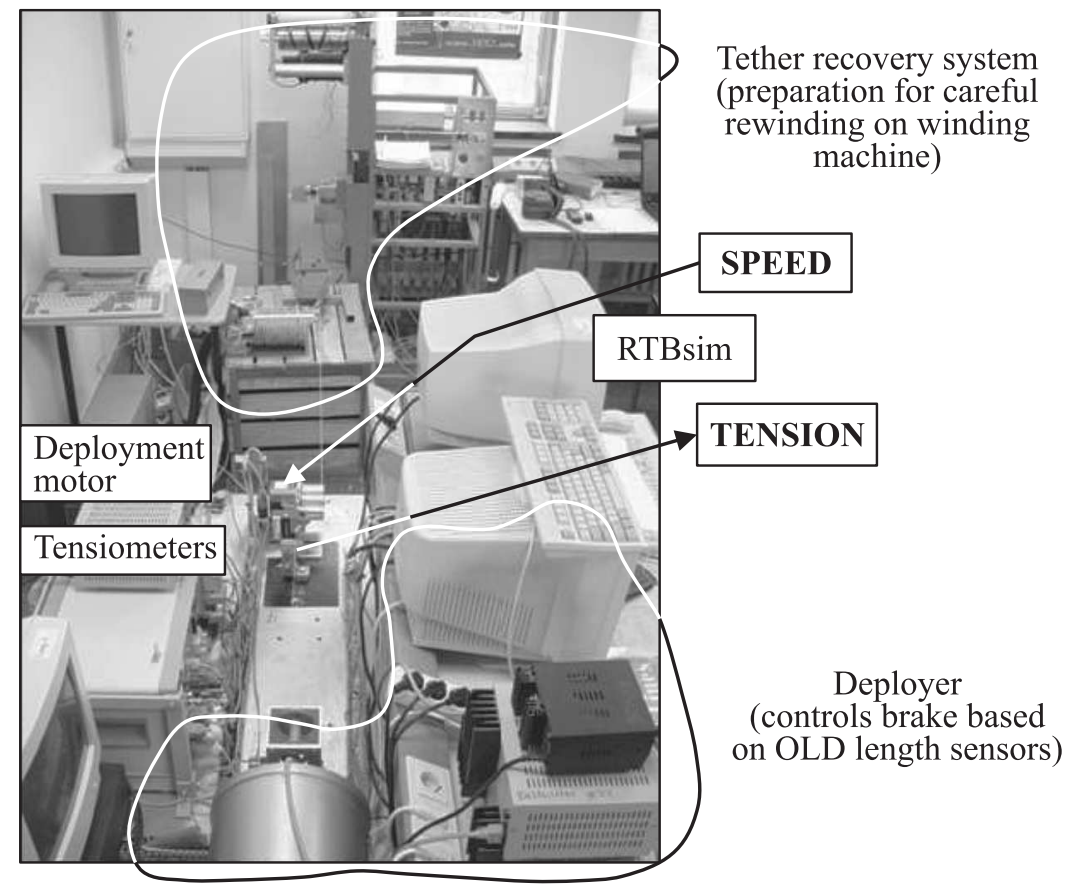

Figure 10 Deployer and test rig layout/architecture 
3. The OBC registers the deployment by receiving the first signals from the OLD length sensors that sense the passing of a tether loop. It then commences its main deployment control.

4. The following control loops occurs during the duration of the test:

(a) the OBC uses the length measurements from the OLD sensors to determine whether tension should be added or subtracted (in order to follow the predefined deployment profile);

(b) it then controls the turns around the barberpole brake accordingly;

(c) fully independently, the test rig measures this change in tension and feeds it to a Real-time Tether Bead Simulator (RTBSim) which is based on MTBSim (see [3] for description);

(d) RTBSim iterates the equations of motion with the new applied tension;

(e) the new deployment velocity is sent from RTBSim to the rig;

(f) the rig tells the pulling motor to 'pull' the tether at the new speed; and

(g) the process repeats.

\section{Limitations}

- Libration angle: The applied tension does not include brake system exitfriction effects due to the tether libration angle (since there is no capacity to simulate the angle in the rig). However, this effect is fairly small compared with other fluctuations in the system $(<30 \%)$.

- Test environment (mechanical): The tests are performed in a pressurized environment at room temperature. The impact of the space environment (vacuum, microgravity, and thermal) has been investigated separately in testing for the YES2 $[3,8]$. The result was an increased variability in delivered friction by the barberpole tension controller. However, the feedback controller allows for inaccuracies in the applied tension. Note also that the gravity-orientation of the canister did not appear to affect the average tension level during deployment.

\subsection{Test Results}

\subsubsection{YES2 deployment testing}

Deployment control using described hardware feasibility has been convincingly demonstrated with the YES2 test campaign that included testing of YES2's two stages of deployment $(3.5$ and $30 \mathrm{~km})$ and induced disturbances to test for robustness [3]. Those disturbances were successfully controlled away by the onboard software and barberpole brake system. Typical results are provided in Fig. 11. 


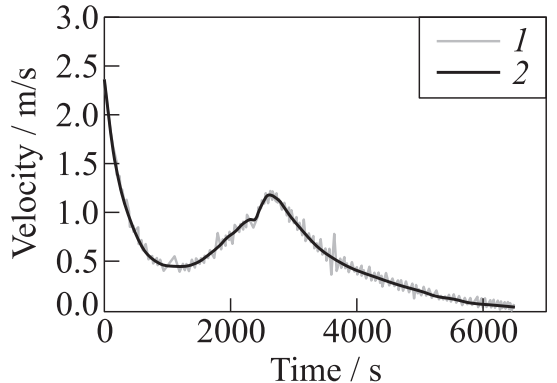

(a)

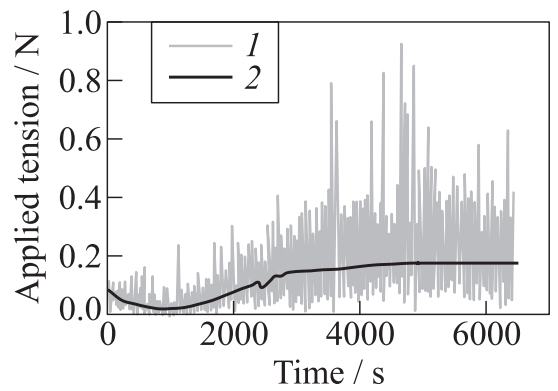

(c)

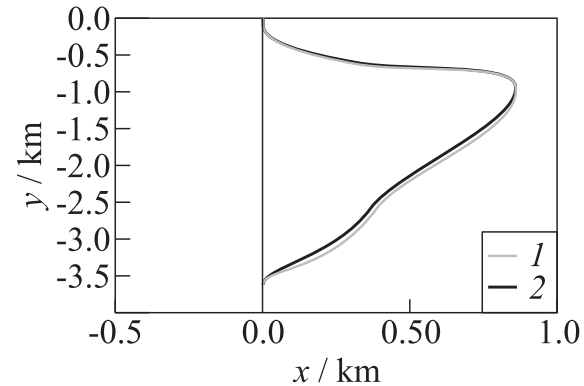

(b)

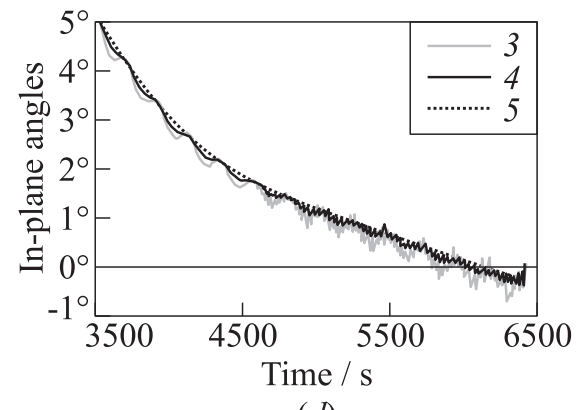

(d)

Figure 11 YES2 first stage deployment results. Actual deployment velocity remains very close to the target path $(a)$, leading to a deployment trajectory in the real-time space simulator in-the-loop that is also very close to actual $(b)$. The tension that the barberpole applies is noisy, but follows well the nominal profile $(c)$. The resulting lateral tether oscillations are also simulated as a result of the applied control and these oscillations are small: the tether remains straight $(d): 1$ - reference; 2 - actual; 3 quarter-tether; 4 - mid-tether; and 5 - end-mass

\subsubsection{Adaptations and partial test for T-Series}

In terms of the mission and hardware functionality, there are two main differences between the YES2 breadboard deployer and that proposed for T-Series.

1. The tether diameter is 0.5 instead of $0.4 \mathrm{~mm}$. This may slightly affect the friction coefficient between the tether and the pole.

2. The deployable tether length (due to canister size) is only $\sim 30 \mathrm{~km}$ instead of the required $100 \mathrm{~km}$. This was solved by 'scaling' the length and velocity of the reference profile so that the deployer would attempt to follow a scaled profile but still deliver the correct tension. With lower speeds, the barberpole will have to create more turns to deliver the same tension. On the test rig side, the simulation is made assuming no scaling but the calculated velocity 
is sent to the rig in scaled form. This way the rig deploys at a rate of 0.3 times that of the simulator.

The T-Series requires much larger tensions and therefore torques on the test rig. For this reason, a different type and stronger motor was installed.

During a trial of the upgraded test rig, a small part of the T-Series deployment was demonstrated (see Fig. 12d). The tracking of the velocity and length profiles was near-perfect (Figs. $12 a$ and 12c) but there was a deviation from the target in-plane angle trajectory. In YES2 testing, it was found that if the target velocity/length profile is approximately followed, then the in-plane trajectory is also followed [3]. However, Fig. $12 b$ clearly shows deviation from the target trajectory around the perigee $(\sim 2000 \mathrm{~s})$ where air drag becomes significant. Since the continuously higher drag acts as a forcing function about the nominal trajectory and since the in-plane state is not measured by the deployer system, the error is unrecoverable. From several more trials, a trend was discovered indicating that an underdeployment (tether too short) leads to a higher in-plane angle at perigee pass, whilst overdeployment leads to a lower angle. Physically, this may be because there is slightly less gravity gradient forces to counteract the perigee airdrag - which acts to push the tether away from the vertical but this is yet to be confirmed.

The 'scaled' nature of this test is best illustrated by Fig. $12 a$. The simulator begins with the correct initial conditions (i.e., $1.33 \mathrm{~m} / \mathrm{s}$ ejection) but commands a smaller rig-speed by factor 0.3 . The OLDs sense an ejection of $0.4 \mathrm{~m} / \mathrm{s}$ which corresponds with its stored target trajectory. It continues to apply the reference tension (which is unscaled) augmented with feedback based on deviations from the length/velocity trajectory. Reference length and velocity are linearly scaled by 0.3 . Tension is maintained constant. Turns are recalculated based on the scaled length and velocity. In-plane angle is unchanged but is not known or used by the OBC in tracking the target trajectory. The feedback gains are not scaled down because it is believed that robustness of the real hardware is a fixed value rather than a fixed length/velocity proportion.

The deployer breadboard OLDs perform well in matching the rig-motor speed (as measured by highly accurate encoders). Furthermore, the software filters the speed very effectively to remove the high-speed noise (see curve 1) which is physically present due to motor speed oscillations below $0.4 \mathrm{~m} / \mathrm{s}$. Such oscillations are a result of a limitation of the motor system. The oscillations do not appear to affect the test, however, since the average speed per second (which is sent to the simulator) is unaltered as is the OLD's filtered velocity. The tension levels will also fluctuate but this averaged out over $1 \mathrm{~s}$ before being sent to the simulator.

The test tension fluctuations are due in part to noise in the tensiometer signals but also from physical effects: Ref. [3] describes how the tension varies significantly over one cycle of unwinding (which equals 4-7 spool tether loops - 


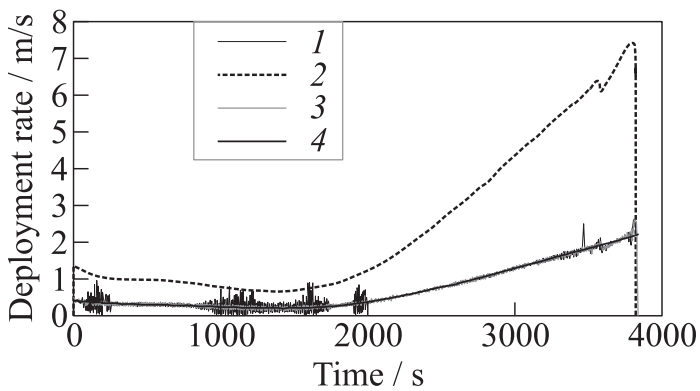

(a)

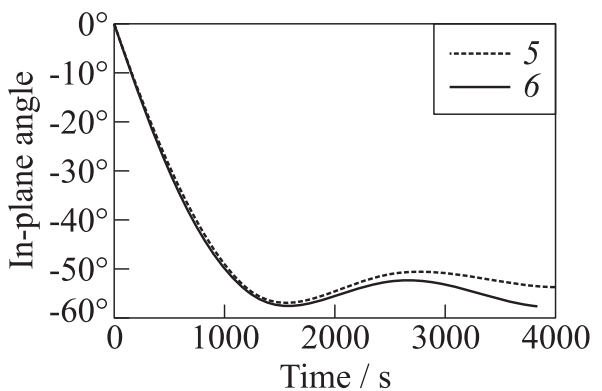

(b)

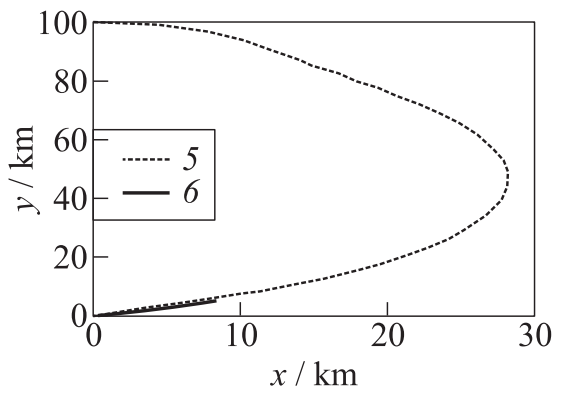

(d)

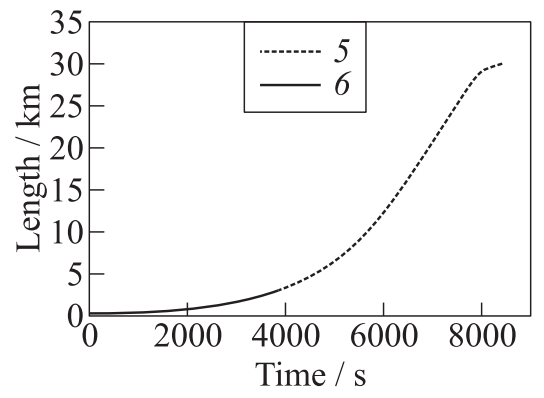

(c)

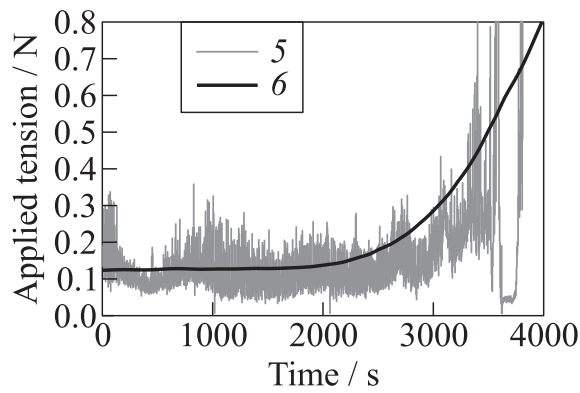

(e)

Figure 12 T-Series demonstration test trial \#1 (test no. 033) (a) velocity profile (1 - rig velocity; 2 - simulator velocity; 3 - OLD-measured velocity; and 4 target (reference) trajectory); $(b)$ in-plane angle profile; $(c)$ length profile; $(d)$ in-plane end-mass track; and $(e)$ applied tension profile $(5$ - reference; and 6 - actual)

depending on winding pattern). The changing equilibrium of the tether distribution on the pole also causes tension fluctuations.

The presence of a stagnant tether strand going out the canister exit hole, to simulate the Kapton-secured canister coupling system (see section 6), did not visibly effect the deployment of the spool proper. Despite cyclical rubbing of the 
deploying tether on the stagnant strand, there was no discernible effect on the tension profile.

\section{CONCLUDING REMARKS}

T-Series is a competitive upper stage option for small solid-booster launchers that aim to:

- circularize $150 \mathrm{~kg}$ payload in LEO; and

- deorbit the launcher stage (in accordance with new guidelines).

Rather than an additional liquid stage, an approximately 160-kilometer tether is attached to the second solid stage. Limitations that apply are an initial perigee altitude of no less than $175 \mathrm{~km}$ - the lower limit is given by air drag effects on the tether - and an initial apogee that is equal to the target altitude lessened by a fraction of the nominal tether length. This tether performs the full function of a liquid stage and is competitive because of:

- good reliability $(96 \%)$;

- lower system mass $(\sim 30 \%)$;

- lower cost (much simpler system); and

- higher accuracy (by tether control based on insertion error).

The primary driver of the tether mission design is the accuracy of the solidstage-delivered orbit. More inaccurate insertion into elliptical orbit quite simply means a longer spool in order to control for the error. Fortunately, the selected tether density is quite low and therefore, the spool mass eats into only a small fraction of the payload. But the tether length is limited by airdrag issues (a long tether not only raises the payload but also lowers the deployer system deeper into the atmosphere), and this consequently limits the correctable solid launcher dispersions to about $40 \mathrm{~km}$ at $3 \sigma$. From a system's viewpoint, the major development issues are thermal control during the deployment end-brake phase, the use of a new tether material (Zylon, capable to withstand high temperatures during braking), and the optimization of the P2 structure to accommodate the multiple canister deployer design. However, these problems are all deemed solvable without substantial difficulty. With regards to the reliability of a tether upper stage, the required mission success rate of $95 \%$ (defined for the study) can be achieved.

Hardware tests to prove the viability of the proposed deployment system for T-Series were partially performed. During the YES2 deployment testing, the hardware and control functionality was demonstrated. In order to demonstrate T-Series specific dynamics, the principle of a scaled-down test was demonstrated 
effectively but as yet only a partial deployment test was performed. The highspeed/high-tension regime was not yet tested. A test of the full deployment must be completed before the T-Series concept can be properly evaluated.

Still, the test already provides some significant findings. The deployment system is able to follow a predefined length and velocity profile to within reasonable accuracy ( $\sim \pm 3 \%$ length, $\sim \pm 10 \%$ velocity). It is thought that a nonscaled test would result in reduced errors, since past experience with the deployer breadboard has shown that the absolute value of the error is reasonably constant and is not proportional to velocity. The scaled tests have the same absolute error but with a smaller velocity/length.

The most startling finding was that the in-plane angle profile appears to be sensitive to small deviations in the length and velocity profiles, which may be related to air drag effects and is to be studied further. There could be severe implications for the T-Series mission because the deviation may tend to grow with deployment time due to the air drag forcing function and a lack of feedback of in-plane state. Other mechanisms may be at work here though, since this behavior was not observed in offline T-Series Monte Carlo mission simulator that usually match very well the test results [9]. In the worst case, a GPS-based feedback of in-plane state (deployment angle) may be required.

\section{REFERENCES}

1. Carroll, J. 1993. SEDS deployer desigh and flight performance. AIAA Space Programs and Technologies Conference and Exhibit September, Huntsville.

2. Willekens, P., A. van Dijk, M. Kruijff, and E. J. van der Heide. 2004. Young Engineers' Satellite, Educational Demonstration of SpaceMail. ESA's "on Station" 17.

3. Hyslop, A., C. Menon, and M. Kruijff. 2005. Simulating space tether deployment on Earth for the YES2 satellite. IAC-05-A2.1.09. 56th Astronautical Congress (International). Fukuoka.

4. Ockels, W. J, E. J. van der Heide, and M. Kruijff. Space Mail and tethers, sample return capability for Space Station Alpha. Oslo. IAF-95-T.4.10.

5. Kruijff, M., E. J. van der Heide, F. De Venuto, M. Dobrowolny, and G. Vannaroni. 2001. Long term stability of bare conductive tethers: Combined results from plasma chamber tests and advanced simulations. STAIF 2001. Albuquerque.

6. Kruijff, M., E. J. van der Heide, and S. Calzada. 2003. YES2 inherently-safe tethered re-entry mission and contingencies. IAF Bremen. IAC-03-IAA.6.2.02.

7. Van der Heide, E. J., M. Kruijff, D. Raitt, and F. Hermanns. 2003. Space spin-in from textiles: Opportunities for tethers and innovative technologies. IAF Bremen. IAC-03-U.2.b.09.

8. Menon, C., M. Kruijff, A. Vavouliotis, A. Miaris, V. Kostopoulos, and F. Angrilli. A European barberpole mechanism for space tether deployment. IAC-05-C2.P.02.

9. Kruijff, M., and E. J. van der Heide. 2001. Integrated test rig for tether hardware, real-time simulator and control algorithms: Robust momentum transfer validated. STAIF 2001. Albuquerque. 